\title{
Author Correction: Robust memristors based on layered two-dimensional materials
}

Miao Wang, Songhua Cai, Chen Pan, Chenyu Wang, Xiaojuan Lian, Ye Zhuo (1), Kang Xu, Tianjun Cao, Xiaoqing Pan, Baigeng Wang, Shi-Jun Liang, J. Joshua Yang (1), Peng Wang (1D) and Feng Miao (1)

Correction to: Nature Electronics https://doi.org/10.1038/s41928-018-0021-4, published online 5 February 2018.

In the version of this Article originally published, the author Xiaoqing Pan's two affiliations with the University of California, Irvine, were mistakenly omitted. They are: Department of Chemical Engineering and Materials Science, University of California, Irvine, CA, USA; Department of Physics and Astronomy, University of California, Irvine, CA, USA. These have now been included in the Article.

Published online: 5 March 2018

https://doi.org/10.1038/s41928-018-0044-X 